\title{
Bergsoniana
}

\section{Bergson et son temps}

\section{Frédéric Worms}

\section{OpenEdition}

Journals

Édition électronique

URL : https://journals.openedition.org/bergsoniana/491

DOI : 10.4000/bergsoniana.491

ISSN : 2800-874X

\section{Éditeur}

Société des amis de Bergson

\section{Référence électronique}

Frédéric Worms, «Bergson et son temps », Bergsoniana [En ligne], 1 | 2021, mis en ligne le 01 juillet 2021, consulté le 08 novembre 2021. URL : http://journals.openedition.org/bergsoniana/491 ; DOI : https://doi.org/10.4000/bergsoniana.491

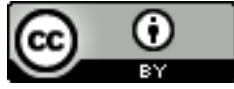

Les contenus de la revue sont mis à disposition selon les termes de la Licence Creative Commons Attribution 4.0 International. 


\title{
BERGSONET SON TEMPS 1
}

\author{
Frédéric WORMS
}

Dans une enquête sur les relations des philosophes à «leur» temps, entendu comme l'époque historique où ils vivent et écrivent, le " présent » en général, la place de Bergson est une des plus paradoxales. Dans son cas, pourrait-on dire, la chose est vite résumée : il n'y a tout simplement pas de telle relation. Ce serait plutôt comme un face à face. D'un côté, dès son premier livre ou presque, il y eut la réception de Bergson par ses contemporains, et qui le placèrent vite au centre de leur temps, définissant même ce dernier en grande partie par lui et par son ouvre. Et combien d'historiens le firent ensuite, sur le moment et jusqu'à aujourd'hui ! "Bergson et son temps, » de ce point de vue, c'est une question de cours, avec ses passages obligés, la querelle de la philosophie nouvelle, la réception dans le monde entier, Proust, Péguy, Jaurès, Sorel, et puis aussi Freud, Einstein, et tant d'autres. Certains ont même été tentés de réduire Bergson à sa " gloire " et de l’y enfermer. Mais lui, de son côté, il l'avait refusé d'avance. Cette pensée du temps se refuse à s'inscrire dans son époque. Philosophe de la durée, mais résistant au temps social, il écrit L'intuition philosophique en $1908 \mathrm{au}$ sommet de cette " gloire » et pour s'opposer à cette réduction. L'intuition est singulière, et il faut la dire intemporelle au sens de sa relation à l'histoire : "Si Spinoza avait vécu avant Descartes, il aurait sans doute écrit autre chose que ce qu'il a écrit mais, Spinoza vivant et écrivant, nous étions sûrs d'avoir le spinozisme tout de même " (Bergson 2011, 8). La seule

1. Le présent article développe de manière entièrement nouvelle certains des éléments proposés au colloque organisé à Cambridge par Matyáš Moravec ainsi que dans une postface au volume édité par Caterina Zanfi : La pensée et le mouvant; Introduction (2020). Je les remercie l'un et l'autre très vivement, ainsi que toutes celles et tous ceux qui sont avec eux au cœur de la relance des études bergsoniennes aujourd'hui, notamment autour des Bergsoniana, et du projet du «Global bergsonism. " 
contemporaine que la philosophie revendique, pour vérifier son intuition, c'est la science. Mais l'époque, l'esprit du temps, il n'en est pas question. C'est l'affaire des salons, du bavardage, bref de l'« homo loquax. » Ainsi, on aurait d'un côté un Bergson "de son temps ", mais malgré lui, et de l'autre Bergson lui-même, refusant de se "situer " dans son époque et de penser un temps qui n'est justement pas le "sien ", même et surtout au prisme de la durée. Pourtant, autre chose s'est finalement produit, qui change tout, et qui fera l'objet de nos brèves indications ici.

Car tout se passe comme si Bergson, lui-même, avait été finalement conduit, nous dirions presque contraint, à aller plus loin, nous indiquant du même coup non seulement quel fut son rapport le plus profond à "son " temps, mais aussi ce que signifie ce lien quand il s'agit d'une véritable relation, et qui vaut pour nous aujourd'hui, tout autant que jamais. $\mathrm{Ce}$ lien avec notre présent va d'ailleurs, lui aussi, plus loin qu'on ne croit. Car, les deux fois où, d'après nous, Bergson a affronté presque malgré lui le défi de "son temps", il a aussi deux fois indiqué le défi que nous découvrons être à nouveau le nôtre. Ce fut, à chaque fois, et par-delà le malentendu qu'ils ont suscité sur le moment, dans ses deux derniers livres. Et sur des questions qui reviennent aujourd'hui au cœur de notre présent : le temps de la vérité, et l'histoire du vivant. Comment n'y reviendrions nous pas, pour comprendre son temps et le nôtre ? Même en quelques mots, cela nous semble nécessaire, et urgent.

\section{Le temps de la vérité}

La première fois où Bergson affronta le défi de son temps ce fut bien sûr dans son tout dernier livre, La Pensée et le mouvant, publié en 1934 mais achevé, on le sait, avec son Introduction, dès 1922. Car ce recueil n'est pas seulement, comme on le crut alors (et encore aujourd'hui, en se fondant sur une lecture sommaire dès son titre), la répétition de sa thèse centrale sur le rapport de la pensée « au » mouvant, ou au temps. C'est, au contraire, une réflexion nouvelle, et d'une importance cruciale, sur la façon dont cette pensée (comme toute pensée) s'inscrit elle-même dans le temps, et dans l'histoire, sur les résistances qu'elle y rencontre, et sur l'effort à produire pour y répondre.

Il est vrai qu'on peut s'y tromper et, de ce point de vue, on peut même dire, comme pour les lignes de train, qu'un malentendu " peut en cacher un autre ». C'est vrai surtout de la thèse capitale sur le «mouvement rétrograde du vrai » ou « l'illusion rétrospective » qui est l'apport nouveau et décisif 
de la première partie de l'Introduction ${ }^{2}$ écrite spécialement pour le livre. Le malentendu le plus simple, mais déjà bien réducteur, est lié à la forme du texte, qui est lui-même une rétrospection, qui plus est à la première personne, Bergson revenant sur son ouvre et sur son parcours! Plutôt que de s'étonner et de se demander : comment le philosophe de la durée, qui avait interdit toute reconstitution de la création, en vient-il à raconter au passé sa propre découverte? Que veut-il nous dire sur l'importance même de la rétrospection, pour confirmer et en fait pour prouver la philosophie? Au lieu de poser ces questions, qui iraient d'emblée au cœur du sujet et conduiraient vers le centre de ce texte, c'est comme si on le banalisait d'avance : ce serait un testament répétitif et non pas une épreuve de vérité ! Mais c'est justement tout l'inverse!

L'autre malentendu est déjà plus profond, on peut même le nommer sans ironie " le malentendu de Jankélévitch ». En quoi consiste-t-il ? Eh bien à concevoir le " mouvement rétrograde du vrai » comme une "illusion » en un sens simplement négatif ou critique. Jankélévitch ne cesse d'y insister, et dès son livre de 1931, ce qui l'a conduit à croire avoir anticipé sur Bergson même : d'après lui, ce mouvement rétrograde n'est que source d'erreur. "Considérée dans toute son ampleur, écrivait-il dans son Bergson, l’illusion rétrospective est une fiction dont on ne saurait exagérer l'importance sociale et la désastreuse ténacité. C'est, pourrait-on dire, 'l'idole' par excellence " (Jankélévitch 1932, 36). ${ }^{3}$ Et il est certain qu'il y a là du vrai. Mais pourquoi alors Bergson parlerait-il du mouvement rétrograde, justement, «du vrai » et comme son critère même?

Le texte, encore lui-même plein de possibilités nouvelles, de l'Introduction à La Pensée et le mouvant, le disait pourtant clairement, comme d'ailleurs les grandes explications rétrospectives de Bergson sur la liberté elle aussi si mal comprise dans sa pensée. Certes, il faut critiquer la "logique de rétrospection ", en tant qu'elle prétend reconstruire le présent avec des éléments du passé. Mais il faut inévitablement s’en servir, justement, rétrospectivement, pour éclairer le passé à la lumière du présent et plus encore pour vérifier l'idée présente par l'éclairage qu'elle donne sur le passé et par ce que l'on peut appeler son effet de vérité. Que le réel « crée du

2. Nous renvoyons aux deux éditions récentes de ce texte d'abord dans l'édition critique de La pensée et le mouvant, publiée aux PUF dans la collection Quadrige (Bergson 2009), et pour la première fois de ce texte de manière spécifique éditée par Caterina Zanfi (Bergson 2020). La pagination dans cette deuxième édition renvoie aussi à celle de la première.

3. Le texte de cette première édition est daté de 1930 dans son avant-propos. 
possible » au lieu d'en résulter, ce n'est pas seulement un acte soudain, qui s'oppose à une illusion de notre esprit : c'est aussi sa fécondité et sa preuve même. C'est par la richesse de ce qui apparaît rétrospectivement que nous mesurons la puissance d'une vérité, de même que c'est par ce qu'il nous apprend sur nous-mêmes, sur la manière dont il nous impliquait tout entier, que nous mesurons la liberté de notre acte. Ou encore sur la manière dont il nous révèle Shakespeare lui-même et son temps, que nous mesurons la puissance de son œuvre! Ce temps n'aurait pas pu le produire et c'est une erreur de le croire ; mais qu'il puisse rétrospectivement le confirmer, dans sa révélation inattendue et infiniment féconde, c'est sa vérité même et il est nécessaire de l'établir. C'est pourquoi Bergson conclut le grand paragraphe sur la logique de rétrospection avec ces mots :

Il ne s'agit certes pas de renoncer à cette logique ni de s'insurger contre elle. Mais il faut l'élargir, l'assouplir, l'adapter à une durée où la nouveauté jaillit sans cesse et où l'évolution est créatrice (Bergson 2009, $19 ; 2020,71)$.

De ce point de vue, tout se retourne et ce n'est pas seulement l'Introduction à La Pensée et le mouvant, avec son aspect parfois défensif (pour montrer comment ses propres thèses ont fini par s'imposer rétrospectivement : « de ces premières réflexions sortirent des conclusions qui sont heureusement devenues presque banales " (Bergson 2009, 19 ; 2020, 72), mais tout le livre, en acte, qui est un effort, par Bergson lui-même, pour inscrire son œuvre dans son temps, pour en prouver la vérité, par ses effets et ses propres évolutions.

Que fait Bergson en effet dans ce recueil ? Deux choses, simultanément : étudier la nature temporelle de la pensée elle-même, et de la vérité, en mesurant la tension entre ces deux aspects qu'on oppose trop souvent de manière simpliste, et aujourd'hui encore. Mais aussi, construire en acte la vérité temporelle de sa propre pensée, par la composition de son recueil. Et par ces deux tâches il répond à "son temps " et cherche à s'y inscrire, en résistant à une réception superficielle, et en assumant cependant de chercher à s'y situer. Ce n'est pas le lieu d'y insister en détail, mais redisons un mot de chacun de ces aspects.

La relation du temps et de la vérité d'abord. Elle repose sur le fait que la durée n'est pas seulement un objet pour la pensée, et contradictoire avec la forme habituelle de celle-ci chez les humains. Mais qu'elle est aussi son moteur interne et vital, lorsque précisément la pensée fait effort pour atteindre le réel, cherche ensuite à le prouver, et aussi à y agir, pour le transformer. Chacun des textes du recueil, nous l'avons montré déjà, est 
ainsi une étude du mouvement de, et dans, la pensée humaine. Notre pensée est elle-même un acte vital. Cela pourrait menacer, croient certains, sa puissance de vérité, son rapport au monde ou au réel. Mais c'est tout le contraire, montre Bergson, puisqu'elle dépasse l'obstacle qui nous en sépare et se prouve par ses effets rétrospectifs en théorie et ses avancées pratiques dans l'histoire. De ce point de vue, la relation avec le texte sur William James, à la fin du recueil, est bien sûr essentielle.

Mais justement, toute la composition du recueil devient alors lumineuse. Il faut partir de la rétrospection. Il faut passer par les grands textes sur la pensée philosophique. Il faut, surtout, circonscrire ensuite l'Introduction à la métaphysique qui fut en 1903 le sommet de la " querelle " et en fait du malentendu du bergsonisme et après laquelle la pensée de Bergson a si profondément changé ! De ce point de vue une Introduction chasse l'autre et on ne s'en est toujours pas réellement aperçu, il serait temps! Enfin, on peut se rattacher rétrospectivement à trois maitres récents qui composent un prisme historique et une confirmation philosophique : Claude Bernard, William James, et Ravaisson. Quoi de plus clair ? Bergson ne sculpte pas ici sa statue, ni ne rédige un testament. Mais il répond à son temps et par sa philosophie du temps, enfin confrontée à la question de l'histoire, et capable de l'assumer. Peu l'ont vu, Henri Marrou, Raymond Aron, mais c'est que, justement, le malentendu avec l'époque avait eu lieu, et s'était encore creusé. En un sens, Bergson l'avait laissé croître trop longtemps. Au moment où il cherche à le lever, il semble se replier sur lui.

Mais ce sera plus vrai encore du deuxième défi qu'il a pourtant si fortement affronté, qui a donné lieu à plus de malentendus encore, mais qui est aussi plus important et plus urgent encore, s'il se peut, pour nous, aujourd'hui.

\section{L'histoire du vivant}

Combien y aura-t-il encore de travaux sur "Bergson et son temps " qui oublieront le texte où Bergson a finalement affronté son temps, son époque, et tenté de la décrire et de la situer dans une histoire étendue au vivant et au monde? Combien faudra-t-il encore de temps avant qu'on lise sérieusement ce texte qui constitue le dernier chapitre de son dernier livre, en 1932 ? Faudra-t-il attendre longtemps encore avant qu'on confronte le diagnostic de Bergson sur le vivant, la technique, la démocratie, avec le défi qui est le nôtre, en ce $21^{\text {ème }}$ siècle, et où il dessine une alternative cruciale, jusque dans le passage d'une ère de surconsommation à une ère de "frugalité " ? N'est-il pas temps, enfin, de revenir à ces pages que l'on 
oublie encore presque tout le temps, et ne serait-ce que pour les discuter ou les contester s'il le fallait ? Oui.

Mais il est vrai que Bergson ne nous a pas facilité la tâche et que, plus encore dans La Pensée et le mouvant, il a presque camouflé la portée de cette confrontation avec l'histoire, comme s'il y avait été contraint, alors même que, bien sûr, elle ne résulte aucunement des seules circonstances, mais aussi de la nécessité de sa propre pensée.

Le texte auquel nous renvoyons ici comme à un des diagnostics les plus importants d'un philosophe sur "son temps, " qui se trouve être encore ou être redevenu le nôtre, voici en effet, on s'en souvient, comment Bergson l'a intitulé : "Remarques finales " avec un sous-titre qui renvoie aux thèses principales du livre dont c'est le dernier chapitre : "Mécanique et mystique ", dans Les Deux sources de la morale et de la religion, donc, en 1932. C'est comme si, vraiment, l'essentiel avait été dit, avec les grandes thèses sur la morale ouverte et close, la religion statique et dynamique, et la science ou la technique entre les deux. Et le dernier chapitre qui, en l'absence de la grande réforme mystique et politique, traite de l'état du monde et de l'histoire du vivant, ce ne serait que des "remarques finales ", supplémentaires et accessoires. C'est, à nouveau, paraître y arriver par la contrainte et préparer, malgré soi, le malentendu. Il ne faudra pas s'étonner, ensuite, si celui-ci survient, aggravé par le décalage de la parution du livre avec « son temps " qui a si profondément changé, entre temps.

Ce n'est pas le lieu, dans cette brève étude indicative, de revenir en détail sur l'ensemble de ces enjeux, que l'on a déjà étudiés ailleurs pour certains d'entre eux et qui, heureusement, sont au cœur des débats présents sur Bergson et même du projet sur le " bergsonisme global "! Nous nous permettrions d'ailleurs, à ce sujet, de renvoyer à la discussion que les responsables de ce projet nous ont permis d'avoir, autour de ce texte, avec l'un des maîtres de l'histoire globale du vivant aujourd'hui, Dipesh Chakrabarty (Chakrabarty et Worms 2020). Nous insisterons donc seulement sur la source du malentendu qui persiste, et l'importance de le dépasser.

Cette source est simple à indiquer en effet : c'est la philosophie des Deux sources de la morale et de la religion elle-même, y compris dans son rapport à l'histoire ; c'est donc, en apparence, sa propre philosophie de l'histoire qui masque ici le rapport de Bergson à l'histoire, à son temps et au nôtre. De fait, tout se passe comme si, pour Bergson, il y avait d'un côté une " nature " humaine, "statique " pour l'essentiel malgré des changements apparents, y compris sociaux, religieux, scientifiques et techniques, et 
une " histoire " qui ne peut dès lors se définir que par la rupture radicale avec cette nature. C'est tout ou rien, en quelque sorte comme d'autres diraient, et au fond au même sens, c'est la totalité, d'un côté, que peut seule dépasser une ouverture radicale, et l'infini. D'un côté la nature close, avec son obligation " morale " dont la guerre révèle toujours la clôture, la fonction «fabulatrice " qui vient la soutenir avec des dieux dont l'évolution dans une fausse histoire revient toujours aux mêmes mythes primitifs et défensifs, et même la technique, en apparence, ne fait que tourner dans ce cercle de l'espère, au risque de l'aggraver en lui donnant les moyens de tout détruire. De l'autre côté donc, une ouverture morale, mais si rare et profonde, même si elle évoque des échos en chacun de nous, qu'elle suppose un saut radical en dehors et au-dessus de l'humain. Les grands mystiques, définis à des degrés divers par leur refus de la clôture et leur appel moral, jusqu'à la réforme absolue pour Bergson du christianisme des Évangiles. Tel est le grand tableau des trois premiers chapitres du livre. Quel décalage, non seulement avec les philosophies de l'histoire qui apparaissent dans les années 1930, entre deux guerres mondiales, mais aussi, justement, avec le diagnostic de l'époque auquel Bergson semble se risquer comme à regret et par défaut, dans le dernier chapitre du livre!

Pourtant, l'ambition du dernier chapitre du livre n'est pas seulement, comme le diront les dernières lignes, trompeuses à cet égard, de pallier l'absence du mystique et de l'ouverture absolue par des solutions secondaires :

A défaut d'une réforme morale aussi complète il faudra recourir aux expédients, se soumettre à une 'réglementation' de plus en plus envahissante, tourner un à un les obstacles que notre nature dresse contre notre civilisation. Mais, qu'on opte pour les grands moyens ou pour les petits, une décision s'impose (Bergson 2013, 338).

Il pourrait bien sembler, alors, que tout ce chapitre ne porte que sur ces expédients, ces " petits moyens " face à un problème qui remonterait luimême à une " nature " immuable et non pas à une situation historique. L'histoire, une variable de la nature ? Et la démocratie ou la justice, un substitut de mystique?

Il faut s'opposer absolument à cette lecture si partielle des "Remarques finales " et voir au contraire à la fois dans le diagnostic de Bergson sur son temps, en 1932, et dans la réponse ou l'alternative qu'il y oppose une thèse deux fois radicale qui doit nourrir le débat sur notre propre époque, aujourd'hui. Mais nous ne pouvons en dire que quelques mots ici.

Tout se joue très précisément sur la signification que Bergson donne au moment même où il écrit, à l'étape de l'histoire où l'humanité est parvenue. 
Cette étape se caractérise très simplement : par la profondeur où elle replace l'espèce humaine dans l'histoire du vivant. C'est ce qui se joue, en fait, par où on retrouve l'alternative morale fondamentale qui, elle aussi, surgit $d u$ vivant et même, dirions-nous pour notre part, du cœur du vivant humain, dans l'histoire du vivant ! C'est donc bien là que tout se joue et dont nous pourrions repartir aujourd'hui comme si nous pouvions et devions reprendre aujourd'hui ce livre par la fin : partir du diagnostic historique sur le vivant, pour y retrouver l'alternative morale et politique et non pas l'inverse!

Ce que montre Bergson avec la plus grande précision en effet, c'est que l'espèce humaine a atteint, avec sa technique qui extrait une énergie inouïe de la Terre, une capacité inédite à se détruire, retrouvant ainsi les guerres les plus archaïques et exterminatrices de son histoire :

Au train dont va la science, le jour approche où l'un des adversaires, possesseur d'un secret qu'il tenait en réserve, aura le moyen de supprimer l'autre. Il ne restera peut-être plus trace du vaincu sur la terre (Bergson 2013, 305).

Mais ce qu'il montre aussi, c'est qu'il existe un idéal d'affranchissement des humains par la technique et la démocratie qui peut orienter l'histoire tout autrement et qui même, une fois surgi, nous fait lire l'histoire autrement par l'illusion rétrospective que nous connaissons bien, désormais ! Ce fut " un souffle démocratique qui poussa en avant l'esprit d'invention " (Bergson 2013, 328) dit Bergson au seuil de cette page, et en assumant justement, comme nous savons aussi, l'effet rétrospectif de cette invention moderne, qui retentit en arrière sur toute l'histoire de l'humanité !

La rétroactivité du présent est à l'origine de bien des illusions philosophiques. Nous nous garderons donc d'attribuer aux quinzième, seizième et dix-huitième siècles $[\ldots]$ des préoccupations démocratiques comparables aux nôtres $[\ldots]$ Il n'en est pas moins vrai que la Réforme, la Renaissance et les premiers syndromes de la poussée inventive sont de la même époque (Bergson 2013, 328-329).

Ainsi, la contemporanéité de la démocratie et de la technique dans sa part créatrice et libératrice est une nouveauté dont la vérité se prouve, cependant, par sa profondeur historique rétroactive !

Certes, Bergson dans la même page reliera cette double poussée démocratique et inventive à l'amour mystique qui dépasse les limites de l'humain. Mais si on y voit une tension qui surgit depuis l'intérieur même de l'humain et du vivant, nous voici au seuil de notre propre temps, et non plus par un supplément à un grand livre sur la morale et la religion, sur le 
clos et l'ouvert, mais plutôt comme à son origine, son commencement, son recommencement ou sa reprise.

Oui, la preuve que Bergson a finalement affronté son temps c'est qu'il nous conduit ou reconduit au seuil du nôtre. C'est une confirmation et une obligation qui ne nous permettent plus d'attendre. On se souviendra donc aussi de ce qu'il disait dans une lettre du 31 Juillet 1940 à Brunschvicg alors que la guerre qu'il avait prédite avait commencé à se déchaîner :

[D] epuis plusieurs années déjà je voyais venir ce qui est arrivé ; mais la réalité s'est chargée de dépasser ce que la fantaisie la plus sombre aurait pu imaginer. Nous avons touché le fond de l'abîme. Du moins savons-nous maintenant où était le mal. Répétons-nous, jour et nuit, le mot de l'empereur romain : 'Laboremus !' (Bergson 2002, 1665). ${ }^{4}$

Travaillons, donc, puisque désormais l'orientation ne fait plus de doute.

\section{Bibliographie}

Bergson, Henri. 2002. Correspondances. Édité par André Robinet. Paris : Presses universitaires de France.

Bergson, Henri. 2009. La Pensée et le mouvant. Paris : Presses universitaires de France.

Bergson, Henri. 2011. L'Intuition philosophique. Paris: Presses universitaires de France.

Bergson, Henri. 2013. Les Deux sources de la morale et de la religion. Paris : Presses universitaires de France.

Bergson, Henri. 2020. La Pensée et le mouvant; Introduction. Paris : Desclée de Brouwer.

Chakrabarty, Dipesh et Frédéric Worms. 2020. "History of Life and Vital Dilemmas. "Filmed November 2020 as part of the first Global Bergsonism conference (held online). https://www.youtube.com/ watch?v=wAHzudad02w.

Jankélévitch, Vladimir. 1932. Bergson. Paris : Félix Alcan.

4. Lettre du 31 Juillet 1940. 
Frédéric Worms est professeur d'histoire de la philosophie contemporaine à l'École normale supérieure de Paris, où il est directeur adjoint du département des Lettres et directeur du Centre international d'études de la philosophie française contemporaine (CIEPFC). Il est membre du Comité consultatif national d'éthique. Il a dirigé l'édition des œuvres de Bergson aux PUF et il est auteur de Bergson ou les deux sens de la vie (PUF, 2004), La philosophie du $X X^{e}$ siècle en France. Moments (Gallimard, 2009), et de récents ouvrages sur le soin et les relations vitales et morales aujourd'hui. Il est fondateur et Président d'honneur de la Société des amis de Bergson.

Résumé : Dans La Pensée et le mouvant, Bergson n'affronte pas seulement le problème fondamental de sa philosophie : celui du rapport de la pensée au temps ou à la durée, et les diverses solutions qu'il lui a données à travers son ouvre. Il étudie également l'effet du temps et de la durée au sein de la pensée, et les nouveaux problèmes qu'il permet d'énoncer, et de résoudre. Ainsi, Bergson nous montre comment ajuster notre pensée, qui est principalement spatiale, à la réalité de la durée et du changement. Bien sûr, depuis son manifeste philosophique Introduction à la métaphysique (au cœur du recueil d'essais) jusqu'à ses derniers essais, l'attitude de Bergson change : il va conférer une valeur de réalité plus forte à la matière, et une valeur de vérité à l'intelligence analytique et à la science, tout en réservant une position privilégiée à l'intuition. Mais il est aussi confronté à de nouveaux problèmes liés à la technique et à la démocratie, concernant les manières concrètes de penser et d'agir dans la durée. Il nous conduit ainsi à la connaissance rétrospective du temps et de l'histoire, dont la vérité ne peut cependant être pleinement préservée, et comprise, qu'en replaçant l'espèce humaine dans l'histoire du vivant.

Mots-clés: Henri Bergson, durée, vérité, philosophie de l'histoire.

\begin{abstract}
In The Creative Mind (La pensée et le mouvant in the original French), Bergson not only confronts the fundamental problem in his philosophy: that of the relationship of thought to time or duration, and the various solutions he gave to it through his work. He also studies the effect of time and duration within thought, and the new problems it allows to state, and to solve. Thus, Bergson shows us how to adjust our thought, which is mainly spatial, to the reality of duration and change. Of course, from the philosophical manifesto Introduction to Metaphysics (at the heart of the collection of essays) to his last essays, Bergson's attitude changes: he will confer a stronger value of reality to matter, and a value of truth to analytic intelligence and science yet reserving a privileged position to intuition. But he also faces new problems pertaining to technology and democracy, concerning the concrete ways of thinking and acting in duration. It thus leads us to the retrospective knowledge of time and history, whose truth can nevertheless be preserved, and fully understood only by replacing the human species in the history of the living.
\end{abstract}

Keywords: Henri Bergson, duration, truth, philosophy of history. 